\title{
Inmunidad Celular en Ganado Vacuno Lechero Naturalmente Infectado con Fasciola hepatica en Cajamarca, Perú
}

\author{
Cellular Immune Response in Dairy Cattle Naturally Infected with \\ Fasciola hepatica in Cajamarca, Peru \\ Heber Silva-Díaz ${ }^{1,3}$, Cristian Hobán-Vergara ${ }^{1}$, Rosmery Cruz-Cerna ${ }^{1}$, \\ Hugo Solana ${ }^{2}$, Pedro Ortiz-Oblitas ${ }^{1}$
}

\section{Resumen}

La fasciolosis producida por Fasciola hepatica es una importante enfermedad parasitaria de la ganadería en muchos países. En Cajamarca, Perú, se reportan prevalencias superiores al $80 \%$ en el ganado lechero. El objetivo del estudio fue evaluar la capacidad de proliferación y la expresión de citoquinas (IFN- $\gamma$ e IL-4) en células mononucleares de sangre periférica de terneras y vacas naturalmente infectadas, contra el antígeno no específico fitohemaglutinina y los antígenos específicos de excreción-secreción del estadio inmaduro y maduro de $F$. hepatica. La capacidad proliferativa fue determinada por linfoproliferación in vitro y la expresión de citoquinas se evaluó en sobrenadantes de cultivo celular por la técnica de ELISA. Las vacas infectadas con $F$. hepatica mostraron una disminución de la capacidad de respuesta frente a estímulos proliferativos inespecíficos y específicos. En el perfil de citoquinas contra el estímulo específico se encontró una baja expresión de IFN- $\gamma$, mientras que la respuesta de IL-4 fue alta, lo que indica que la respuesta permanece polarizada hacia una respuesta de tipo Th2. En el análisis comparativo, las terneras expresaron niveles de IFN- $\gamma$ más altos que los valores obtenidos en las vacas, pero con una expresión similar de IL-4. No se observaron diferencias en la respuesta inmune a los antígenos del estadio inmaduro y maduro del parásito. Los resultados demuestran que a medida que la enfermedad progresa se expresa una respuesta inmunomoduladora en los animales adultos naturalmente infectados con $F$. hepatica.

Palabras clave: Fasciola hepatica, respuesta inmune celular, infección natural, ganado vacuno lechero, Cajamarca

${ }^{1}$ Facultad de Ciencias Veterinarias, Universidad Nacional de Cajamarca, Cajamarca, Perú

${ }^{2}$ Facultad de Ciencias Veterinarias, Universidad Nacional del Centro de la Provincia de Buenos Aires, Argentina

${ }^{3}$ E-mail: h.silvadiaz@hotmail.com

Recibido: 23 de febrero de 2015

Aceptado para publicación: 25 de mayo de 2015 
Fasciolosis caused by Fasciola hepatica is an important parasitic disease of livestock in many countries. In Cajamarca, Peru, prevalence rates above $80 \%$ are reported in dairy cattle. The aim of the study was to assess the proliferative capacity and expression of cytokines (IFN- $\gamma$ and IL-4) in peripheral blood mononuclear cells from cows and calves against nonspecific phytohemagglutinin and specific excretory-secretory antigens from immature and mature stages of $F$. hepatica. The proliferative capacity was determined by in vitro lymphocyte proliferation and the cytokine expression was evaluated in cell culture supernatants by ELISA. Cows infected with $F$. hepatica showed a decreased capacity to respond against nonspecific and specific proliferative stimulus. In the cytokine profile against specific stimulus, IFN- $\gamma$ expression was low while IL-4 response was high, indicating that the response remains polarized towards a $\mathrm{TH} 2$ type response. Naturally infected calves expressed higher IFN- $\gamma$ levels compared to cows, with a similar expression of IL-4 between both groups of animals. No differences were observed in the immune response to antigens of immature and mature stages of the parasite. The results show an immunomodulatory response in adult animals that are naturally infected with $F$. hepatica as the disease progresses.

Key words: Fasciola hepatica, cellular immune response, natural infection, dairy cattle, Cajamarca

\section{INTRODUCCIÓN}

La infección por Fasciola hepatica es considerada en el Perú como una enfermedad infecciosa parasitaria emergente en salud pública (Marcos et al., 2007). Asimismo, es la zoonosis helmíntica más importante en varias regiones del mundo, afectando principalmente a humanos, bovinos, ovinos y caprinos (Robinson y Dalton, 2009). La enfermedad humana y animal está distribuida en el $71 \%$ del territorio peruano y es endémica de la sierra (Claxton et al., 1997; Marcos et al., 2004; Valencia et al., 2005; Lopez et $a l ., 2012)$. En la provincia de Cajamarca, donde la industria lechera es una actividad económica importante, la prevalencia en el ganado bovino es superior al 75\% (SENASA, 2007).

En zonas endémicas del Perú, la enfermedad en el ganado constituye un significativo impacto económico, con pérdidas no menores a los US\$50 millones al año (Espinoza et al., 2010). Estas pérdidas son principalmente por disminución de la producción lechera y de la fertilidad, menor ganancia de peso, decomiso de órganos en los centros de beneficio y costos en el tratamiento antiparasitario. La fasciolosis en el ganado bovino lechero en Cajamarca se controla principalmente por quimioterapia y apoyada con actividades de manejo agropecuario; sin embargo, el problema persiste y empeora, debido a la resistencia que ha mostrado el parásito a dosis estándares de triclabendazol, el antihelmíntico más usado contra esta parasitosis (Ortiz et al., 2013).

Aunque el uso de una vacuna es una estrategia que podría controlar eficazmente el problema de la fasciolosis en Cajamarca, todavía no ha sido posible su desarrollo, debido a vacíos de conocimiento sobre las características de la respuesta inmunitaria del hospedero a la infección (Ortiz et al., 2000) y se carece de estudios que permitan entender la respuesta inmunitaria celular en ganado con 
infección natural. Por tal motivo, el presente trabajo evaluó la capacidad de proliferación y la expresión de citoquinas (IFN- $\gamma$ e IL-4) en células mononucleares de sangre periférica (PBMC) de terneras y vacas infectadas naturalmente, frente a un antígeno no específico, la fitohemaglutinina (PHA) y frente a dos antígenos específicos de excreción/secreción del estadio inmaduro (FhESPI) y maduro (FhESP-M) de $F$. hepatica.

\section{Materiales y Métodos}

\section{Ubicación}

El presente estudio fue realizado en el valle de Cajamarca, Perú, y en el Laboratorio de Inmunología e Investigación de la Facultad de Ciencias Veterinarias de la Universidad Nacional de Cajamarca (UNC).

\section{Animales y Diseño Experimental}

Dieciocho animales hembras de raza Holstein fueron seleccionados de varios establos lecheros, donde 12 animales estaban infectados naturalmente con $F$. hepatica. Las terneras tenían entre 4 y 8 meses de edad y las vacas, entre 2 y 6 años. La infección fue demostrada por estudio coproparasitológico (técnica de sedimentación rápida) e inmunológico (inmunoblot). Muestras de sangre fueron obtenidas de cada animal por punción de la vena yugular en tres oportunidades, usando tubos heparinizados de extracción al vacío.

\section{Preparación de Antígenos}

FhESP-M fue obtenido de especímenes maduros de $F$. hepatica, recuperados desde conductos biliares de hígados infectados co-

Se aplicó el siguiente diseño experimental con seis animales por grupo:

\begin{tabular}{|c|c|c|}
\hline Grupos & Antígenos & Evaluaciones \\
\hline Terneras infectadas (TI) & \multirow{3}{*}{$\begin{array}{l}\text { Cultivo celular de PBMC } \\
\text { estimuladas con FhESP- } \\
\text { M, FhESP-I y PHA }\end{array}$} & \multirow{3}{*}{$\begin{array}{l}\text { - } \text { Capacidad linfoproliferativa } \\
\text { - Expresión de citoquinas } \\
\text { (IFN- } \gamma \text { e IL-4) }\end{array}$} \\
\hline Vacas infectadas (VI) & & \\
\hline Control no infectado $(\mathrm{CNI})^{1}$ & & \\
\hline
\end{tabular}

PBM C: células mononucleares de sangre periférica

FhESP-M : antígeno específico de excreción-secreción del estadio maduro de F. hepatica FhESP-I: antígeno específico de excreción-secreción del estadio inmaduro de F. hepatica PHA: antígeno inespecífico fitohemaglutinina (mitógeno)

${ }^{1}$ Compuesto por terneras y vacas

lectados en el camal local; mientras que FhESP-I fue obtenido desde especímenes inmaduros de 28 días de la infección, recuperados del parénquima hepático de cuyes (Cavia porcellus).

La obtención de los antígenos requirió del cultivo de los especímenes y posterior centrifugación del sobrenadante, para lo cual se siguió el protocolo descrito por Ortiz et al.
(2000), con ligeras modificaciones (en vez de utilizar un espécimen maduro por $3 \mathrm{ml}$ de medio se usó $1 \mathrm{ml}$ y $0.5 \mathrm{ml}$ de medio RPMI 1640 para el estadio maduro e inmaduro, respectivamente; además los especímenes solo se incubaron por 12 horas).

La concentración proteica de los antígenos fue determinada con el kit comercial Quick Start ${ }^{\mathrm{TM}}$ Bradford Protein Assay 
(Bio-Rad®) por espectrofotometría molecular y varió desde 500 a $1000 \mu \mathrm{g} / \mathrm{mL}$. El antígeno inespecífico (mitógeno) fue PHA comercial (Sigma Aldrich ${ }^{\circledR}$ ).

\section{Aislamiento de PBMC}

Las PBMC fueron recuperadas desde sangre periférica por centrifugación de gradiente de densidad en Histopaque 1077 (Sigma Aldrich®). La viabilidad celular fue determinada en cámara de Neubauer, previa coloración con azul de tripán $0.4 \%$, y varió entre 97 y 99\% (Siga Aldrich ${ }^{\circledR}$ ).

\section{Ensayo de Linfoproliferación}

La capacidad linfoproliferativa frente a FhESP-M, FhESP-I y PHA fue evaluada con $5 \mu \mathrm{g} / \mathrm{mL}$, a razón de $2 \times 10^{5}$ células $/ 100 \mu \mathrm{L} /$ pocillo. Posterior a la incubación a $37^{\circ} \mathrm{C}$ y $5 \%$ de $\mathrm{CO}_{2}$ por $72 \mathrm{~h}$, se determinó la proliferación por reacción colorimétrica usando Thiazolyl Blue Tetrazolium Bromide (MTT) comercial (Sigma Aldrich®). La variación de la proliferación fue evaluada por el índice de estimulación.

\section{Expresión de Citoquinas}

Las PBMC fueron cultivadas a razón de $4 \times 10^{5}$ células $/ 200 \mu \mathrm{L} /$ pocillo. Las concentraciones de los antígenos evaluados fueron: PHA $10 \mu \mathrm{g} / \mathrm{mL}$, FhESP-M y FhES-I $10 \mu \mathrm{g} / \mathrm{mL}$ (para IFN- $\gamma$ ), y 10 y $20 \mu \mathrm{g} / \mathrm{mL}$ (para IL-4). La concentración de IFN- $\gamma$ y IL-4 fue determinada en sobrenadantes de cultivo celular por la técnica de ELISA, para los cuales se utilizaron los kits comerciales Bovine IFN Gamma ELISA Reagent Set (Genway Biotech $\left.{ }^{\circledR}\right)$ y Bovine Interleukin-4 ELISA Kit (Bio-Rad®).

\section{Análisis Estadístico}

Se realizó el análisis de varianza y comparaciones múltiples de Tukey para determinar diferencias entre grupos y antígenos en relación a la capacidad proliferativa y expresión de citoquinas. Se consideró significativo un nivel de $\mathrm{p}<0.05$. En el análisis se utilizaron los paquetes estadísticos SPSS 22.0 e Infostat/E.

\section{Resultados}

Las PBMC de terneras y vacas infectadas naturalmente con $F$. hepatica mostraron una disminuida capacidad de respuesta proliferativa inespecífica frente al mitógeno PHA en relación al control no infectado. De manera similar, los animales infectados mostraron una débil respuesta proliferativa a los antígenos específicos de $F$. hepatica (Fig. 1).

El análisis de varianza indicó diferencia estadística entre los promedios de índices de estimulación proliferativa en relación a grupos y antígenos $(\mathrm{p}<0.001)$. No obstante la débil respuesta proliferativa de los grupos TI y VI frente al estímulo inespecífico (PHA), las PBMC del grupo de las TI mostraron una respuesta superior en comparación al de las VI ( $\mathrm{p}<0.05)$. Por otro lado, no se encontró diferencia estadística entre los antígenos específicos en la respuesta proliferativa; sin embargo, ambos indujeron una débil respuesta en comparación al PHA $(\mathrm{p}<0.05)$.

La estimulación con el antígeno inespecífico (PHA) indujo una moderada expresión de IFN- $\gamma$ en todos los animales, siendo más potente en los animales del grupo control CNI que en el de los infectados (Fig. 2). De forma similar, la expresión de IL-4, al usarse PHA como estímulo, fue inducida exitosamente en todos los animales, pero en menor proporción respecto al IFN- $\gamma$ (Fig. 3). Cuando las células fueron estimuladas con antígenos específicos (FhESP), ningún grupo experimental produjo cantidades altas de IFN- $\gamma$ (Fig. 2). Caso contrario sucedió con la expresión de IL-4, donde los animales infectados respondieron con cantidades altas al ser estimulados (Fig. 3).

No obstante la baja expresión de IFN- $\gamma$ de los grupos TI y VI frente al estímulo específico (FhESP), el análisis de varianza mos- 


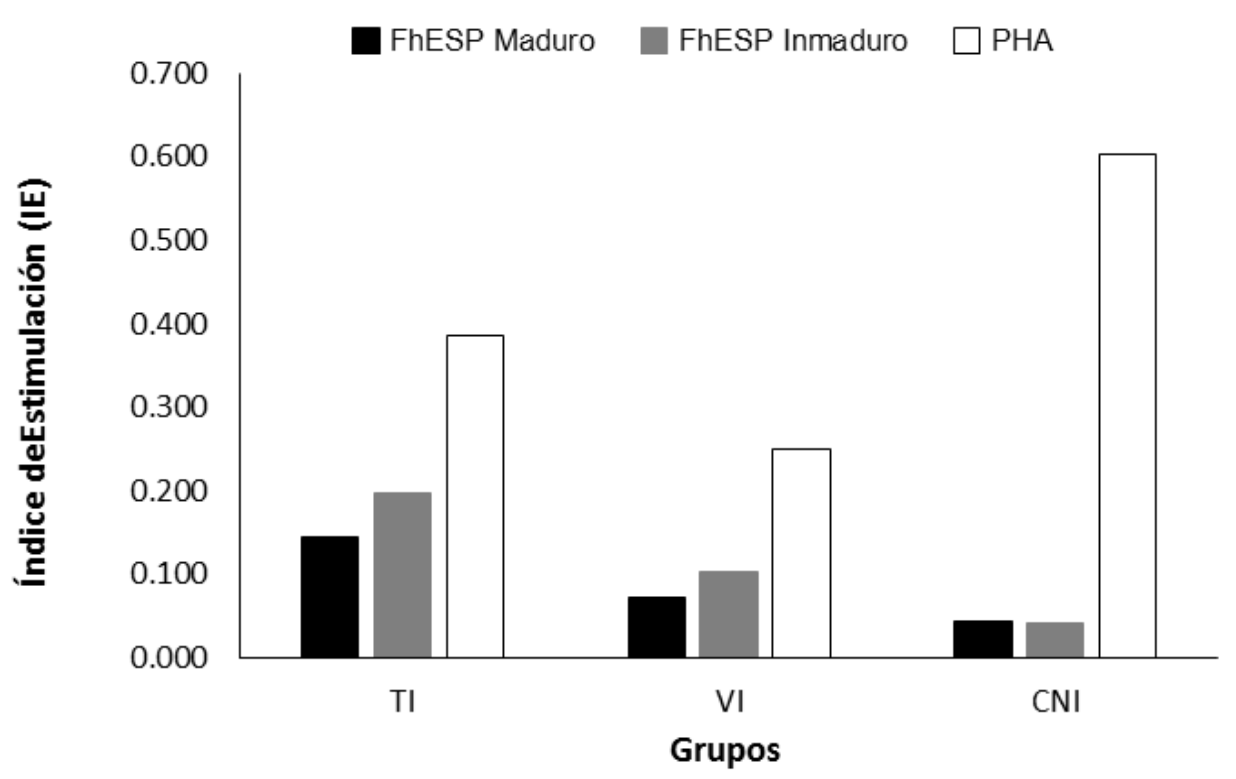

Figura 1. Promedio de los índices de estimulación proliferativa de las células mononucleares de sangre periférica (PBMC) de los animales infectados (terneras [TI] y vacas [VI]) y no infectados (CNI) con F. hepatica, frente a los antígenos FhESP Maduro, FhESP Inmaduro y PHA

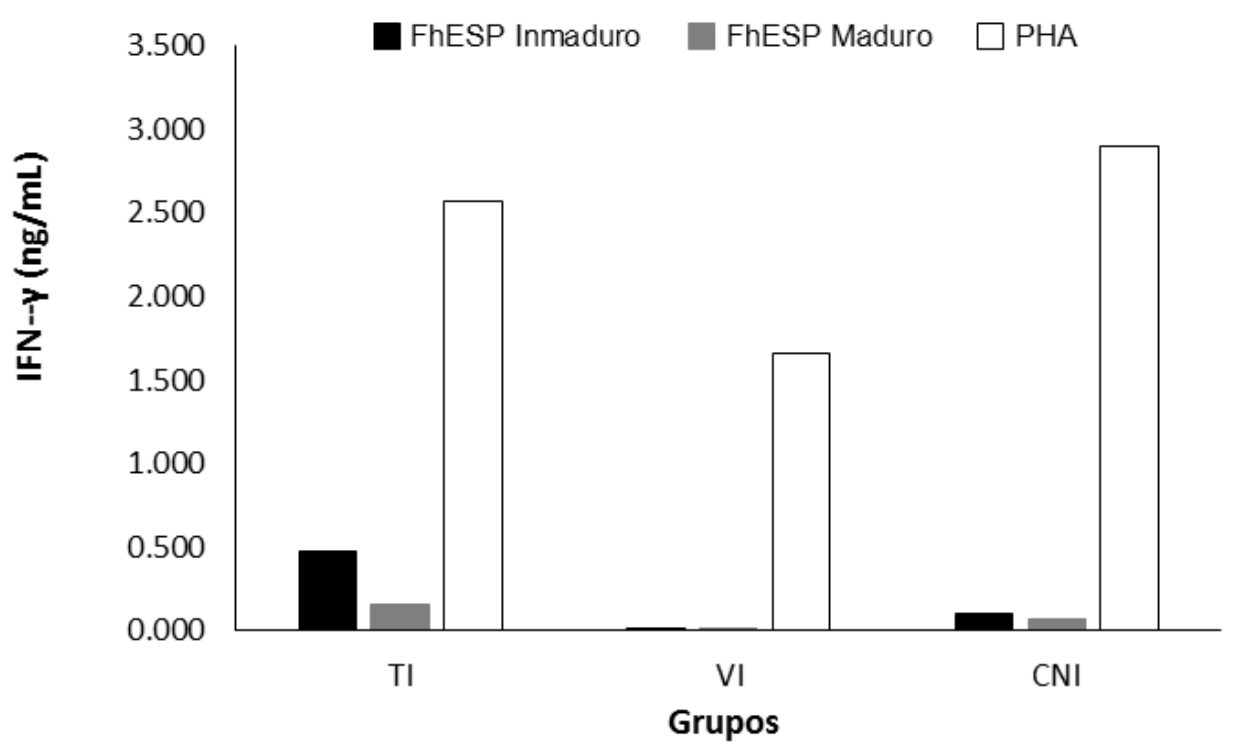

Figura 2. Promedio de la expresión de IFN- $\gamma(\mathrm{ng} / \mathrm{mL})$ de las células mononucleares de sangre periférica (PBMC) de los animales infectados (terneras [TI] y vacas [VI]) y no infectados (CNI) con $F$. hepatica, frente a los antígenos FhESP Maduro, FhESP Inmaduro y PHA 


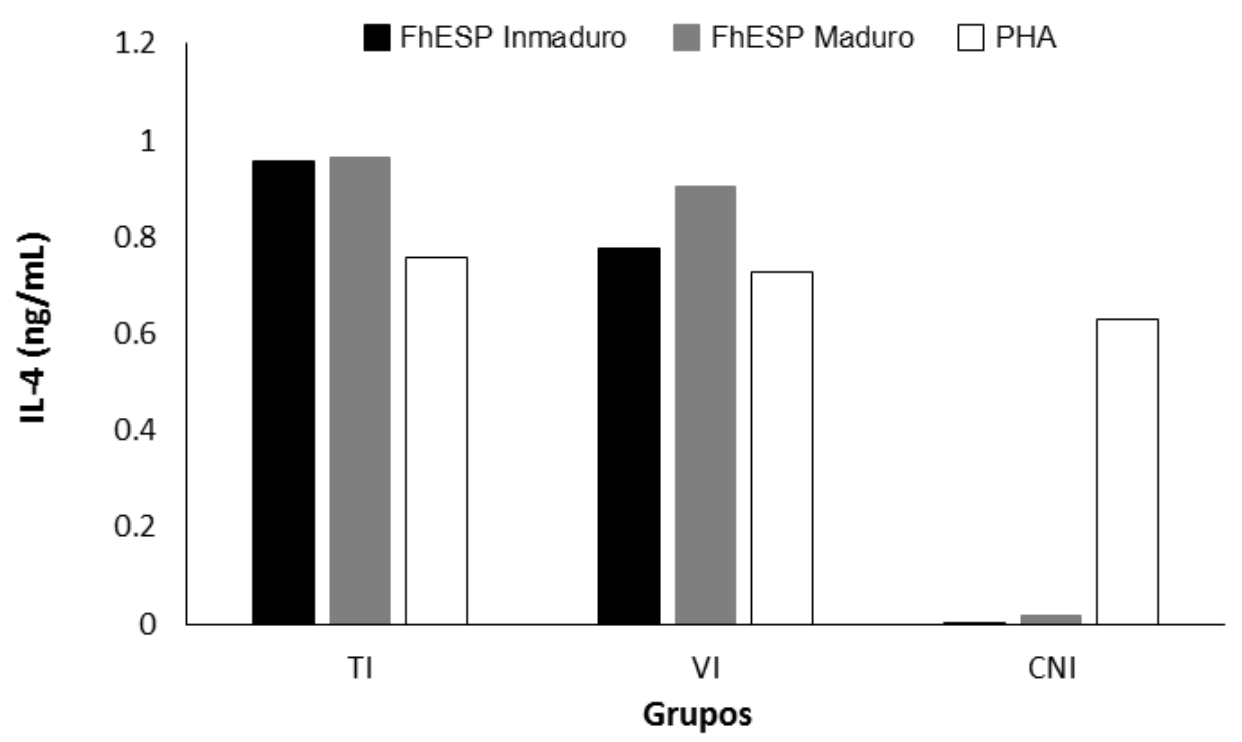

Figura 3. Promedio de la expresión de IL-4 (ng/mL) de las células mononucleares de sangre periférica (PBMC) de los animales infectados (terneras [TI] y vacas [VI]) y no infectados (CNI) con F. hepatica, frente a los antígenos FhESP Maduro, FhESP Inmaduro y PHA

tró diferencia estadística entre los promedios de IFN- $\gamma$ e IL-4 en relación a los grupos y antígenos $(\mathrm{p}<0.001)$. Las PBMC del grupo de las TI expresaron una cantidad significativamente mayor de IFN- $\gamma$ en comparación a las VI $(\mathrm{p}<0.05)$. La expresión de citoquinas fue estadísticamente similar, tanto al usarse los antígenos provenientes de estadios inmaduros y maduros del parásito.

\section{Discusión}

Los resultados de linfoproliferación sugieren un efecto inmunosupresor de la infección por F. hepatica sobre los PBMC de los animales infectados. De hecho, en las TI, debido a la probable infección reciente o cambio de la fase aguda a crónica, el grado de inmunosupresión es menor; mientras que las VI, por su mayor edad, mayor tiempo de enfermedad y resistencia del parásito a ser eliminado con tratamiento farmacológico, se encuentran en fase crónica, en la cual la inmunosupresión es mayor. El efecto inmunosupresor de $F$. hepatica se explica por los múltiples papeles que juegan los antígenos parasitarios en la evasión e inmunomodulación ejercida por el parásito (Flynn et al., 2010).

El efecto de $F$. hepatica sobre la respuesta de las PBMC a mitógenos ha sido estudiada en bovinos (Brown et al., 1994; McCole et al., 1999), caprinos (MartínezMoreno et al., 1999) y ratas (Cervi et al., 2001; Tliba et al., 2002a; Gironenes et al., 2007), poniendo de manifiesto diferencias interespecíficas, así como entre infecciones agudas y crónicas. Así, por ejemplo, en ganado vacuno infectado se produce una elevada respuesta a mitógenos con producción de IFN- $\gamma$ en fases agudas, mientras que en fases crónicas cae tanto la respuesta a mitógenos como la producción de IFN- $\gamma$ (Clery et al., 1996).

Este efecto inmunosupresor en fases crónicas fue observado previamente en en- 
sayos experimentales en cabras (MartínezMoreno et al., 1997; Gironenes et al., 2007) y ratas (Cervi et al., 1998; Gironenes et al., 2007). No obstante, el presente trabajo es pionero en el estudio de la capacidad proliferativa y la expresión de citoquinas en PBMC de ganado vacuno lechero infectado naturalmente con $F$. hepatica en respuesta al mitógeno PHA y antígenos de secreciónexcreción del estadio inmaduro y maduro del parásito.

El efecto inmunomodulador no varió respecto al origen de proteínas de secreciónexcreción (estadio inmaduro o maduro de $F$. hepatica), donde se ha descrito que la composición molecular y su proporción varían según el estadio del parásito (Morphew et al., 2007; Robinson et al., 2009); y que, por lo tanto, las proteínas de secreción-excreción de ambos estadios tienen el potencial de ser inmunomoduladores del sistema inmune del hospedero.

En el presente estudio, tal como se esperaba, los animales controles (CNI) no mostraron respuesta proliferativa a los estímulos específicos por carecer de exposición previa, mientras que los animales infectados (TI y VI) mostraron una respuesta muy baja, siendo las TI las que mostraron una respuesta superior. Estos resultados podrían indicar que las terneras, debido a una infección reciente, aún tienen la capacidad de respuesta proliferativa específica. Sin embargo, el fallo a la respuesta proliferativa en las VI frente a antígenos específicos concuerda con estudios previos en ovinos (Chauvin et al., 1995; Moreau et al., 1998), ratas (Gironenes et al., 2007), bovinos (Bossaert et al., 2000) y caprinos (Martínez-Moreno et al., 1997), lo que confirma el efecto inmunomodulador que ejercen las proteínas de excreción-secreción del parásito sobre el sistema inmunológico del hospedero.

En cuanto a la expresión de citoquinas, el perfil observado con baja expresión de IFN- $\gamma$ y alta de IL- 4 demuestra que la respuesta inmune celular en ganado lechero in- fectado naturalmente con $F$. hepatica está polarizada hacia una respuesta tipo Th2. Asimismo, la mayor expresión de IFN- $\gamma$ en las terneras respecto a las vacas demuestra la inmunomodulación de la respuesta a medida que avanza la enfermedad. Las terneras ,debido a su infección reciente, responden todavía, aunque débilmente, con expresión de IFN- $\gamma$ antes de polarizarse a una respuesta Th2, donde la IL-4 es la dominante.

Estos resultados concuerdan con otros estudios, donde quedó de manifiesto la producción de IFN- $\gamma$ típica de la respuesta Th1 durante las primeras semanas de infección (Clery et al., 1996; Tliba et al., 2002a,b); sin embargo, en etapas crónicas esta producción decae hasta extinguirse, evidenciándose el cambio a una respuesta Th2 con elevada expresión de IL-4 (Brown et al., 1994; O’Neill et al., 2000; Gironenes et al., 2007). No obstante, en un estudio en ovejas cola delgada de Indonesia (ITT) resistentes a infección por F. gigantica (Pleasance et al., 2011) y en otro en conejos con alta protección conferidos con una vacuna contra $F$. hepatica (Espino y Rivera, 2010), se demuestra que una respuesta temprana tipo Th1 o la dominancia de esta está asociada a la protección y resistencia a la infección.

El presente estudio, no obstante, reitera que la respuesta en animales naturalmente infectados está polarizada hacia una respuesta de tipo Th2, lo que evidencia el efecto inmunosupresor e inmunomodulador que ejerce la infección por $F$. hepatica en el hospedero. Estos resultados concuerdan con un trabajo realizado también en ganado lechero infectado natural y crónicamente con $F$. hepatica en Brasil, donde se demostró que las células de tejido hepático de los animales expresaban altas cantidades de IL-4 e IL-10 en relación al IFN- $\gamma$, típica de una respuesta Th2 (Mendes et al., 2013).

Estos resultados también ponen en evidencia que el efecto inmunosupresor e inmunomodulador hacia una respuesta tipo Th2, que induce la infección por $F$. hepatica 
en ganado naturalmente infectado, podría afectar su capacidad para responder normalmente a otras infecciones, principalmente a aquellas que requieren de una potente respuesta tipo Th1, como las producidas por agentes biológicos intracelulares. Esta consecuencia negativa y agravante de la infección por $F$. hepatica ha sido reportada en estudios con animales infectados experimentalmente con F. hepatica y otros agentes infecciosos como Mycobacterium tuberculosis (Flynn et al., 2007), Bordetella pertusis (Brady et al., 1999) y Toxoplasma gondii (Miller et al., 2009).

La capacidad inmunomoduladora de los FhESP sobre el hospedero se explica por la amplia variedad y funciones de las proteínas que los componen (O'Neill et al., 2001; Dalton et al., 2003; Morphew et al., 2007; Robinson et al., 2009; Dowling et al., 2010). Entre estas proteínas, la peroxirredoxina (FhPrx) demostró inducir la activación de los macrófagos por la vía alternativa (AAM Ø) y, esta a su vez, promueve la respuesta Th2 (Donnelly et al., 2005; Robinson et al., 2010). También, la catepsina L1 (FhCL1) ha demostrado que inhibe la activación de los macrófagos por la vía clásica (CAMØ) y suprime el desarrollo de una respuesta Th1 (Hamilton et al., 2009). Asimismo, se ha demostrado, además, que los FhESP inducen propiedades tolerogénicas en células dendríticas mieloides e inhiben la respuesta proliferativa de células esplénicas a mitógenos (Cervi y Masih, 1997; Falcón et al., 2010).

Por otro lado, la expresión de citoquinas no se diferenció al usarse los antígenos provenientes de estadios inmaduros y maduros del parásito, en los que se han descrito diferencias de proporción y composición molecular (Morphew et al., 2007; Robinson et al., 2009). Esto sugiere que tanto el estadio inmaduro que migra a través del parénquima hepático, como el maduro que ocupa los conductos biliares, tienen la capacidad para inmunomodular la respuesta inmune del hospedero a través de la acción de sus proteínas de secreción-excreción.
Los resultados sugieren que la respuesta inmune tipo Th2 en ganado naturalmente infectado con $F$. hepatica, observada previamente en infección experimental, puede también ser responsable del establecimiento de la fase crónica, reinfección y el mantenimiento de la infección natural en ganado que se encuentran en continuo contacto con el parásito.

\section{Conclusiones}

- Vacas y terneras naturalmente infectadas con F. hepatica mostraron una disminuida capacidad linfoproliferativa frente a estímulos inespecíficos (PHA) y específicos (FhESP), poniendo de manifiesto el efecto inmunosupresor de la infección.

- Las vacas infectadas responden con una respuesta inmune polarizada $\mathrm{Th} 2$, con niveles bajos de IFN- $\gamma$ y altos de IL- 4 .

- Las terneras infectadas expresaron niveles más altos de IFN- $\gamma$ en comparación a las vacas, pero similares de IL-4, lo que demuestra la inmunomodulación de la respuesta a medida que avanza la enfermedad.

- Los antígenos específicos del estadio inmaduro y maduro del parásito no mostraron inducir una respuesta inmune diferente.

\section{Agradecimiento}

Los autores agradecen al Consejo Nacional de Ciencia y Tecnología (CONCYTEC) del Perú por el financiamiento del presente trabajo

\section{Literatura Citada}

1. Bossaert K, Jacquinet E, Saunders J, Farnir F, Losson B. 2000. Cellmediated immune response in calves to single-dose, trickle, and challenge infections with Fasciola hepatica. Vet 
Parasitol 88: 17-34. doi: 10.1016/S03044017(99)00200-9

2. Brady MT, O'Neill SM, Dalton JP, Mills KHG. 1999. Fasciola hepatica suppresses a protective Th1 response against Bordetella pertussis. Infect Immun 67: 5372-5378.

3. Brown WC, Davis WC, Dobbelaere DA, Rice-Ficht AC. 1994. CD4+ T-cell clones obtained from cattle chronically infected with Fasciola hepatica and specific for adult worm antigen express both unrestricted and Th2 cytokine profiles. Infect Immun 62: 818-827.

4. Cervi L, Cejas H, Masih DT. 2001. Cytokines involved in the immunosuppressor period in experimental fasciolosis in rats. Int J Parasitol 31: 1467-1473.

5. Cervi L, Masih DT. 1997. Inhibition of spleen cell proliferative response to mitogens by excretory-secretory antigens of Fasciola hepatica. Int J Parasitol 27: 573-579. doi: 10.1016/S0020-7519(96)00188-9

6. Cervi L, Rossi G, Cejas H, Masih DT. 1998. Fasciola hepatica-induced immune suppression of spleen mononuclear cell proliferation: role of nitric oxide. Clin Immunol Immunopathol 87: 145-154. doi:10.1006/clin.1997.4499

7. Chauvin A, Bouvet G, Boulard C. 1995. Humoral and cellular immune responses to Fasciola hepatica experimental primary and secondary infection in sheep. Int J Parasitol 25: 1227-1241. doi: 10.1016/0020-7519(95)00039-5

8. Claxton JR, Zambrano H, Ortiz P, Amorós C, Delgado E, Escurra E, Clarkson MJ. 1997. The epidemiology of fasciolosis in the inter-Andean valley of Cajamarca, Peru. Parasitol Int 46: 281-288. doi:10.1016/S1383-5769(97) 00039-1

9. Clery D, Torgerson P, Mulcahy G. 1996. Immune responses of chronically infected adult cattle to Fasciola hepatica. Vet Parasitol 62: 71-82. doi: 10.1016/0304-4017(95)00858-6
10. Dalton JP, Neill SO, Stack C, Collins P, Walshe A, Sekiya M, et al. 2003. Fasciola hepatica cathepsin L-like proteases: Biology, function, and potential in the development of first generation liver fluke vaccines. Int J Parasitol 33: 1173-1181. doi: 10.1016/S0020-7519(03) 00171-1

11. Donnelly S, O'Neill SM, Sekiya M, Mulcahy G, Dalton JP. 2005. Thioredoxin peroxidase secreted by Fasciola hepatica induces the alternative activation of macrophages. Infect Immun 73: 166-173. doi: 10.1128/ IAI.73.1.166-173.2005

12. Dowling DJ, Hamilton CM, Donnelly S, La Course J, Brophy PM, Dalton J, O'Neill SM. 2010. Major secretory antigens of the helminth Fasciola hepatica activate a suppressive dendritic cell phenotype that attenuates Th17 cells but fails to activate Th2 immune responses. Infect Immun 78: 793-801. doi: 10.1128/IAI.00573-09

13. Espino AM, Rivera F. 2010. Quantitation of cytokine mRNA by realtime RT-PCR during a vaccination trial in a rabbit model of fascioliasis. Vet Parasitol 169: 82-92. doi: 10.1016/ j.vetpar.2009.12.018

14. Espinoza JR, Terashima A, HerreraVelit P, Marcos LA. 2010. Fasciolosis humana y animal en el Perú: impacto en la economía de las zonas endémicas. Rev Peru Med Salud Pública 27: 604-612.

15. Falcón C, Carranza F, Martínez FF, Knubel CP, Masih DT, Motrán CC, Cervi L. 2010. Excretory-secretory products (ESP) from Fasciola hepatica induce tolerogenic properties in myeloid dendritic cells. Vet Immunol Immunopathol 137: 36-46. doi: 10.1016/ j.vetimm.2010.04.007

16. Flynn RJ, Mannion C, Golden O, Hacariz O, Mulcahy G 2007. Experimental Fasciola hepatica infection alters responses to tests used for diagnosis of bovine tuberculosis. Infect Immun 75: 1373-1381. doi: 10.1128/ IAI.01445-06 
17. Flynn RJ, Mulcahy G, Elsheikha HM. 2010. Coordinating innate and adaptive immunity in Fasciola hepatica infection: implications for control. Vet Parasitol 169: 235-240. doi: 10.1016/j.vetpar. 2010.02 .015

18. Gironenes N, Valero MA, GarciaBodelon MA, Chico-Calero I, Punzon C, Presno M, Mas-Comma S. 2007. Immune suppression in advanced chronic fascioliasis: an experimental study in a rat model. J Infect Dis 195: 1504-1512. doi: $10.1086 / 514822$

19. Hamilton CM, Dowling DJ, Loscher CE, Morphew RM, Brophy PM, O'Neill SM. 2009. The Fasciola hepatica tegumental antigen suppresses dendritic cell maturation and function. Infect Immun 77(6): 2488-98. doi: 10.1128/IAI.00919-08

20. Lopez M, White AC Jr, Cabada MM. 2012. Burden of Fasciola hepatica infection among children from Paucartambo in Cusco, Peru. Am J Trop Med Hyg 86: 481-485. doi: 10.4269/ ajtmh.2012.11-0448

21. Marcos LA, Maco V, Terashima A, Samalvides F, Miranda E, Tantaleán M, et al. 2004. Hiperendemicidad de fasciolosis humana en el Valle del Mantaro, Perú: factores de riesgo de la infección por Fasciola hepatica. Rev Gastroenterol Perú 24: 158-164.

22. Marcos LA, Terashima A, Leguía, G, Canales M, Espinoza JR, Gotuzzo E. 2007. La infección por Fasciola hepatica en el Perú: una enfermedad emergente. Rev Gastroenterol Perú 27: 389-396.

23. Martínez-Moreno A, Jiménez-Luque V, Moreno T, Redondo ESH, De Las Mulas JM, Pérez J. 1999. Liver pathology and immune response in experimental Fasciola hepatica infections of goats. Vet Parasitol 82: 19-33. doi: 10.1016/S0304-4017(98)00262-3

24. Martínez-Moreno A, Martínez-Moreno FJ, Acosta I, Gutiérrez PN, Becerra C, Hernández S. 1997. Humoral and cellular immune responses to experimental Fasciola hepatica infections in goats. Parasitol Res 83: 680-686.

25. McCole DF, Doherty ML, Baird AW, Davies WC, McGill K, Torgerson PR. 1999. T cell subset involvement in immune responses to Fasciola hepatica infection in cattle. Parasite Immunol 21: 1-8. doi: 10.1046/j.1365-3024.1999. 00188.x

26. Mendes E, De Olivera T, Lopes $S$, Menezes-Sousa D, Bartholomeu D, Martins I, et al. 2013. Expression of IL-4, IL-10 and IFN- $\gamma$ in the liver tissue of cattle that are naturally infected with Fasciola hepatica. Vet Parasitol 195: 177-182. doi: 10.1016/j.vetpar.2013. 03.035

27. Miller CMD, Smith NC, Ikin RJ, Boulter NR, Dalton JP, Donnelly S. 2009. Immunological interactions between 2 common pathogens, Th1inducing protozoan Toxoplasma gondii and the Th2-inducing helminth Fasciola hepatica. PLoS ONE 4(5). doi: 10.1371/ journal.pone.0005692

28. Moreau E, Chauvin A, Boulard C. 1998. Lack of humoral and cellular responses against glutathione $\mathrm{S}$ transferases in Fasciola hepatica experimentally infected sheep. Vet Res 29:585-591.

29. Morphew RM, Wright HA, LaCourse EJ, Woods DJ, Brophy PM. 2007. Comparative proteomics of excretorysecretory proteins released by the liver fluke Fasciola hepatica in sheep host bile and during in vitro culture ex host. Mol Cell Proteomics 6: 963-972.

30. O'Neill SM, Brady MT, Callanan JJ, Mulcahy G, Joyce P, Mills KHG et al. 2000. Fasciola hepatica infection downregulates Th1 responses in mice. Parasite Immunol 22: 147-155.

31. O'Neill SM, Mills KHG, Dalton JP. 2001. Fasciola hepatica cathepsin L cysteine proteinase suppresses Bordetella pertussis-specific interferon gamma production in vivo. Parasite Immunol 23: 541-547. 
32. Ortiz P, Scarcella S, Cerna C, Rosales C, Cabrera M, Guzmán M, et al. 2013. Resistance of Fasciola hepatica against triclabendazole in cattle in Cajamarca (Peru): a clinical trial and an in vivo efficacy test in sheep. Vet Parasitol 195: 118-121. doi: 10.1016/ j.vetpar.2013.01.001

33. Ortiz PL, Claxton JR, Clarkson MJ, McGarry J, Williams DJL. 2000. The specificity of antibody responses in cattle naturally exposed to Fasciola hepatica. Vet Parasitol 9: 121-134. doi: 10.1016/ S0304-4017(00)00360-5

34. Pleasance J, Wiedosari E, Raadsma HW, Meeusen E, Piedrafita D. 2011. Resistance to liver fluke infection in the natural sheep host is correlated with a type- 1 cytokine response. Parasite Immunol 33: 495-505. doi: 10.1111/ j.1365-3024.2011.01305.x

35. Robinson MW, Dalton JP. 2009. Zoonotic helminth infections with particular emphasis on fasciolosis and other trematodiases. Philos T Roy Soc B 364: 2763-2776. doi: 10.1098/rstb.2009.0089

36. Robinson MW, Hutchinson AT, Dalton JP, Donnelly S. 2010. Peroxiredoxin: a central player in immune modulation. Parasite Immunol 32: 305-313. doi: 10.1111/j.1365-3024.2010.01201.x
37. Robinson MW, Menon R, Donnelly SM, Dalton JP, Ranganathan S. 2009. An integrated transcriptomics and proteomics analysis of the secretome of the helminth pathogen Fasciola hepatica: proteins associated with invasion and infection of the mammalian host. Mol Cell Proteomics 8: 1891-1907. doi: 10.1074/mcp.M900045-MCP200

38. [SENASA] Servicio Nacional de Sanidad Agraria. 2007. Informe Anual. Servicio Nacional de Sanidad Agraria, Cajamarca, Perú. 7 p.

39. Tliba O, Moire N, Vern YLE, Boulard C, Chauvin A, Sibille P. 2002a. Early hepatic immune response in rats infected with Fasciola hepatica. Vet Res 33: 261-270.

40. Tliba O, Sibille P, Boulard C, Chauvin A. 2002b. Early hepatic cytokine mRNA expression in experimental rat fasciolosis. Vet Parasitol 103: 237-249. doi: 10.1016/ S0304-4017(01)00584-2

41. Valencia N, Pariona A, Huamán M, Miranda F, Quintanilla S, Gonzales A. 2005. Seroprevalencia de fasciolosis en escolares y ganado vacuno en la provincia de Huancavelica, Perú. Rev Peru Med Salud Pública 22: 96-102. 